\title{
An Incremental-Selective Cooperative Relaying Scheme with Best Relay Selection in WSN
}

\author{
Shaoqing Wang, Hanlin Yang, Ning Zhou, An Wang \\ The Electronic System Engineering Company of China, Beijing, China \\ Email: 429086198@qq.com
}

Received January 2015

Copyright (C) 2015 by authors and Scientific Research Publishing Inc.

This work is licensed under the Creative Commons Attribution International License (CC BY).

http://creativecommons.org/licenses/by/4.0/

(c) (i) Open Access

\begin{abstract}
In wireless sensor networks, the traditional multi-relay incremental cooperative relaying (MIR) scheme could improve the system throughput over the fading channel enormously by exploiting multiple relay nodes to retransmit the copy of the source packet to the destination in turn, but increase the energy consumption and transmission delay. In order to mitigating the energy consumption and transmission delay, this paper proposes a new cooperative relaying scheme termed as incremental-selective relaying with best-relay selection (ISR), which selects the best relay node from the candidate relays to retransmit the packet to the destination only when the direct transmission between the source and the destination is not successful. Expressions of normalized throughput, normalized delay and energy efficiency for the ISR and MIR systems are derived respectively and their performances are compared through simulations. The results show that normalized throughput, normalized delay and energy efficiency for the ISR system all outperform the corresponding performances of the MIR system. Especially, there are different the optimal number of relays which can maximize the energy efficiency of system.
\end{abstract}

\section{Keywords}

Cooperative Relaying, Wireless Sensor Networks, Normalized Throughput, Delay, Energy Efficiency

\section{Introduction}

Energy-constrained wireless sensor networks (WSN) are composed of nodes powered by batteries, for which replacement or recharging is very difficult, if not impossible [1]. Therefore, minimizing the energy consumption 
for data transmission becomes one of the most important design considerations for WSN. But channel fading has an important effect on data transmission in WSN and increases the required energy consumption under a certain throughput requirement. Cooperative relaying has been considered as a potential candidate to combat the effects of channel fading by exploiting cooperative diversity gain achieved via cooperation among the relays nodes in WSN [2]. The basic idea of it results from the broadcasting property of wireless transmission so that relay nodes can overhear the information from the source node and can then forward what is received to the destination. So, cooperative relaying can provide multiple independently-faded channels for the same information transmission so that the probability that all received signals at the destination suffer deep fading will be significantly reduced. Therefore, from this viewpoint, applying cooperative relaying technique into WSN might mitigate the required transmitting power under a certain throughput requirement and save the energy consumption for data transmission.

A variety of low-complexity cooperative relaying schemes were proposed firstly in [3], which included fixed relaying (FR), selection relaying (SR) and incremental relaying (IR) schemes. However, these cooperative relay schemes base on the basic single-relay cooperation model, which is only composed of one source node, one destination and one relay node. Noting that there are always several candidate relays for the cooperative communication between the source and the destination, a great many of multi-relay cooperative schemes are studied and analyzed in [4]-[6].

However, the advantages of aforementioned cooperative relaying schemes come at the expense of a reduction in the system spectral efficiency since the relays must transmit on orthogonal channels in order to avoid interfering with the source node and with each other as well. For example, the single-relay FR system leads to a certain loss in spectral efficiency because it requires two time phase periods for half-duplex transmission. Moreover, in FR system with M relays, which retransmit the copy of the source packet to the destination in turn, $M+1$ orthogonal channels are employed, which means that the number of required channels, the energy consumption and transmission delay all increase linearly with the number of relays. Therefore, the inefficient use of the channel resources or multi-retransmission in cooperative relaying technique might increase the energy consumption and delay for data transmission in WSN.

This problem of the inefficient use of the channel resources can be eliminated with the use of the best-relay selection scheme, in which the "best" relay node only is selected to retransmit the source packet to the destination [7]. Hence, two channels only are required in this case regardless of the number of relays. The FR and SR schemes with best-relay selection are proposed in [8]-[11] respectively. Moreover, in [7]-[11], the authors showed that this best-relay selection scheme has the same diversity order as the multi-relay cooperative relaying scheme in terms of the capacity outage. Therefore, the efficient resource utilization by the best-relay selection scheme does not sacrifice the signal quality. However, two channels are still required in these schemes.

Different from these schemes, the single-relay IR system performs in full-duplex transmission when the direct transmission between the source and destination nodes is successful and in half-duplex transmission only when the direct transmission is not successful, so IR scheme can increase the resource utilization over FR or SR scheme. Therefore, the IR scheme with best-relay selection can enhance the resource utilization compared with the FR or SR scheme with the best-relay selection. Designing the IR scheme with best-relay selection suitable for WSN is still an open problem.

In this paper, a new cooperative relaying scheme termed as incremental-selective relaying with best-relay selection (ISR) is proposed, which integrated IR and SR strategies proposed in [3]. Different from best-relay selection schemes proposed in [7]-[11], ISR system performs in full-duplex transmission when the direct transmission is successful and selects the best relay node from the relays, which have decoded the source signal successfully, to retransmit the source packet to the destination only when the direct transmission is not successful. Hence, the number of required orthogonal channels in ISR system is one or two. Therefore, ISR scheme can enhance the resource utilization and save the energy consumption compared with best-relay selection schemes proposed in [7]-[11].

We focus on the dual-hop ISR system to study its performance over the Rayleigh fading channels. The main contribution of this paper is the derived expressions of normalized throughput, normalized delay and energy efficiency of the ISR system. Moreover, the expressions of normalized throughput, delay and energy efficiency for the multi-relay IR (MIR) system are also derived. The performance of the ISR and the MIR system is compared through simulations.

The remainder of this paper is organized as follows. Section two introduces the ISR system model. Expres- 
sions of normalized throughput, normalized delay and energy efficiency for the ISR and MIR systems are derived respectively in section three and Section four. Some numerical results are discussed in Section five. Finally, some conclusions are drawn in Section six.

\section{System Model and ISR Scheme}

Consider $M+2$ relevant nodes in a WSN, represented respectively by s (source node), d (destination node), and $M$ available relay nodes $r_{i}(i=1,2 \cdots M)$, and assume that s wants to sent data to d, as illustrated in Figure 1. The time is slotted, and the length of a time slot can satisfy the transmission latency and spread latency of the data packet. The proposed ISR scheme with the best-relay selection acts as follows.

In the first slot, s transmits a data packet to d, where the cyclic redundancy check (CRC) bits of each packet facilitate perfect error detection at the receiving node. Due to the broadcast nature of the wireless medium, all the relay nodes can overhear this data packet. For one case, upon successful reception of the packet at d, it broadcasts an ACK message, and then s transmits a new data packet in the next time slot and all the relays just idle. For the other case, if d receives a packet in error, it broadcasts a NACK message identifying the corrupted packet, and then the best relay node is selected from a set of candidate relays, which has received the packet successfully in the first slot, to retransmit it to $d$ in the second slot. The node d drops the corrupted packets and only decodes based on the retransmitted packet. If D node still fails to receive the retransmitted packet, this packet will be dropped. Moreover, if none of the relays receives the source packet successfully in the first slot, this packet will be also dropped.

Next the wireless channel and packet error rate models are described. Flat quasi-static Rayleigh fading channels are considered for the channel between each two nodes, hence the channel coefficients are assumed to be constant during a complete slot, and can vary from a slot to another independently. Let $h_{s d}, h_{s i}(i=1,2 \cdots M)$ and $h_{i d}$ be the channel coefficient of s-d, s- $r_{i}$ and $r_{i}-d$ channels, which are modeled as zero-mean complex Gaussian random variables with unit variance. Thus, the channel gain, $\left|h_{s d}\right|,\left|h_{s i}\right|$ and $\left|h_{i d}\right|$, is modeled as a Rayleigh random variable. Furthermore the channel gain squared, $\left|h_{s d}\right|^{2},\left|h_{s i}\right|^{2}$, and $\left|h_{i d}\right|^{2}$, is modeled as an exponential random variable with unit mean. The noise term is modeled as zero-mean, complex Gaussian random variables with equal variance $N_{0}$. So, the probability distribution function (PDF) of the instantaneous received SNR $\gamma$ of the links can be written as

$$
f_{\gamma}(\gamma)=\frac{1}{\sigma_{m n}} \exp \left(-\frac{\gamma}{\sigma_{m n}}\right)
$$

where $(m n)$ denotes the different links and $\sigma_{m n}$ is the average SNR of links and can be expressed by

$$
\sigma_{m n}=\frac{P_{t} r_{m n}^{-\alpha}}{N_{0}}
$$

where $P_{t}$ is the transmit power assumed to be constant for all nodes, $r_{m n}$ denotes the distance of links and $\alpha$ is path loss exponent.

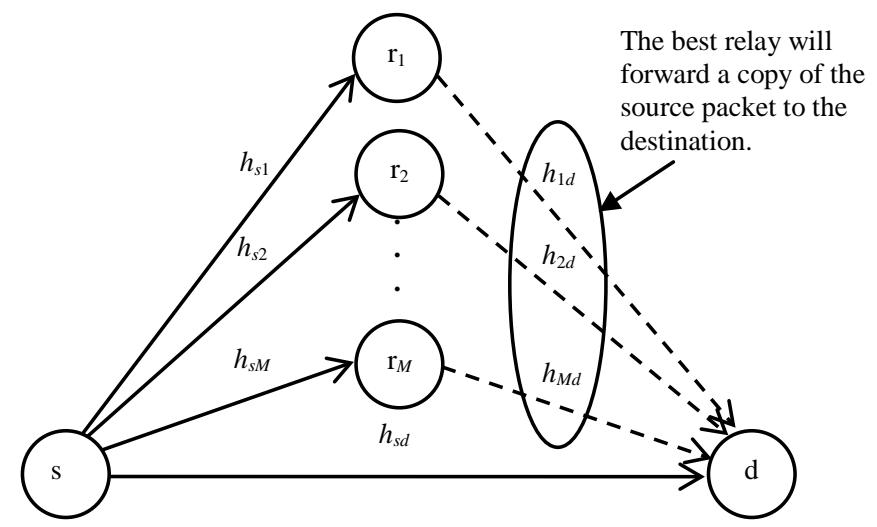

Figure 1. Illustration of the ISR system with the best-relay selection. 
Assuming that uncoded BPSK modulation is adapted in the ISR system, the closed-form expression for the average bit error rate $(B E R)$ of a link is given by

$$
\operatorname{BER}\left(\sigma_{m n}\right)=\frac{1}{2} \sqrt{1-\frac{\sigma_{m n}}{1+\sigma_{m n}}}
$$

For the length of $L$ bits of a data packet, the packet error rate of a link can be obtained as

$$
P E R_{m n}=1-(1-B E R)^{L}
$$

In the ISR system, relays which has received the source packet successfully in the first slot are denoted as the candidate relays, the number of which is assumed to be $k(k=1,2 \cdots M)$. Let $\gamma_{i d}(i=1,2 \cdots k)$ represents the instantaneous SNR of the link between the candidate relay and d. If the instantaneous SNR of some $r_{i}-$ d link is maximal among the set of SNR $\left\{\gamma_{i d}\right\}$, this relay will be is selected to retransmit the source packet to $\mathrm{d}$ in the second slot.

Noting that d drops the corrupted packets and only decodes based on the retransmitted packet, the received SNR at $d$ can be expressed by

$$
\gamma_{\max }=\max \left\{\gamma_{1 d}, \gamma_{2 d}, \cdots \gamma_{k d}\right\}
$$

Using (1), the CDF of $\gamma_{\max }$ can be written as

$$
F_{\gamma_{\max }}(\gamma)=F_{\gamma_{\max }}\left(\gamma_{1 d} \leq \gamma, \gamma_{2 d} \leq \gamma, \ldots, \gamma_{k d} \leq \gamma\right)=\prod_{i=1}^{k}\left(1-\exp \left(-\frac{\gamma}{\sigma_{i d}}\right)\right)
$$

where $\sigma_{i d}$ is the average SNR of the $\mathrm{r}_{i}$-d $(i=1,2 \cdots k)$ link.

Then the PDF of $\gamma_{\max }$ can be found by taking the derivative of (6) with respect to $\gamma$ and can be obtained as

$$
f_{\gamma_{\max }}(\gamma)=\frac{\mathrm{d} F_{\gamma_{\max }}(\gamma)}{\mathrm{d} \gamma}=\sum_{n=1}^{k}(-1)^{n+1} \cdot \sum_{k_{1}=1}^{k-n+1} \sum_{k_{2}=k_{1}+1}^{k-n+2} \ldots \sum_{k_{n}=k_{n-1}+1}^{k}\left\{\prod_{j=1}^{n} \exp \left(-\frac{\gamma}{\sigma_{k_{j} d}}\right) \sum_{j=1}^{n} \frac{1}{\sigma_{k_{j} d}}\right\}
$$

So, the mean value of $\gamma_{\max }$ can be expressed by

$$
E\left[\gamma_{\max }\right]=\int_{0}^{\infty} f_{\gamma_{\max }}(\gamma) r \mathrm{~d} \gamma
$$

Combing (3) and (8), the conditional error rate of the retransmitted packet can be expressed by

$$
\operatorname{PER}\left(\gamma_{\max }\right)=1-\left(1-\operatorname{BER}\left(E\left[\gamma_{\max }\right]\right)\right)^{L}
$$

Using (3) and (9), the average PER of the ISR system can be written as

$$
P E R^{I S R}=P E R_{s d} \prod_{i=1}^{M} P E R_{s i}+P E R_{s d} \cdot \sum_{k=1}^{M}\left\{\left(\begin{array}{c}
M \\
k
\end{array}\right)\left(\prod_{i=1}^{k}\left(1-P E R_{s i}\right)\right) P E R\left(\gamma_{\max }\right) \prod_{i=k+1}^{M} P E R_{s i}\right\}
$$

The first term in the above expression is the event indicating the failure of both the transmission over the s-d channel and the transmissions over all the s-r channels. The second term corresponds to the event indicating the failure of the transmission over the s-d channel while the failure of retransmission with the best relay.

\section{Performance Analysis}

In this section, we characterize the system performance in terms of normalized transmission delay, throughput and energy efficiency.

In the ISR system, if the thing that $d$ receives the source packet in the first slot or the other thing that $d$ fails to receive the source packet and none of relays can receives the source packet in the first slot happens, the packet transmission delay is one slot. In addition, the packet transmission delay is two slots. Therefore, the normalized 
transmission delay of ISR system can be obtained as

$$
T^{I S R}=1 \cdot\left(1-P E R_{\mathrm{sd}}+P E R_{\mathrm{sd}} \prod_{i=1}^{M} P E R_{\mathrm{si}}\right)+2 \cdot P E R_{\mathrm{sd}}\left(1-\prod_{i=1}^{M} P E R_{\mathrm{si}}\right)
$$

Define the system normalized throughput as the packet success rate per the unit of delay. So, using (10) and (11), the system normalized throughput can be written as

$$
G^{I S R}=\frac{1-P E R^{I S R}}{T^{I S R}}
$$

Assume that the total power consumption of the system is composed of the power consumption of power amplifiers of the transmitters and all other circuit blocks of all the nodes. Let $\beta(0<\beta<1)$ denotes the loss factor of the power amplifier and $P_{c t}$ and $P_{c r}$ represent the power consumption of circuit blocks of the transmitter and receiver respectively. The power consumption of the ACK and NACK messages is ignored in this paper. Hence, the total consumed energy of transmitting the same data packet with the ISR scheme can be expressed as

$$
\begin{aligned}
E^{I S R}= & \left(1-P E R_{s d}\right)\left(P_{t}(1+\beta)+P_{c t}+(M+1) P_{c r}\right) L / R_{b} \\
& +P E R_{s d} \cdot \prod_{i=1}^{M} P E R_{s i} \cdot\left\{\left(P_{t}(1+\beta)+P_{c t}+(M+1) P_{c r}\right) L / R_{b}\right\} \\
& +P E R_{s d}\left(1-\prod_{i=1}^{M} P E R_{s i}\right)\left\{\left(2 P_{t}(1+\beta)+2 P_{c t}+(M+2) P_{c r}\right) L / R_{b}\right\}
\end{aligned}
$$

where the first term stands for the energy consumption while the success of the transmission over the s-d channel, the second term represents the energy consumption while the failure of both the transmission over the s-d channel and the transmissions over all the $s-r_{i}$ channels and the third term indicates the energy consumption while the retransmission with the best relay.

Define the number of packets by transmitted successfully per the unit of energy consumption as the energy efficiency of the system. Using (10) and (13), energy efficiency of the ISR system can be written as

$$
\eta^{I S R}=\frac{1-P E R^{I S R}}{E^{I S R}}
$$

\section{Performance Analysis of Multi-Relay IR System}

For the fair compare between the ISR and the Multi-relay IR (MIR) System, still consider $M+2$ relevant nodes in a WSN, represented respectively by s node, $\mathrm{d}$ node and $M$ available relay nodes, and assume that s wants to sent data to d. The MIR scheme acts as follows. In the first slot, s transmits the packet to d and the relays also try to decode this packet. Similarly to the ISR system, relays which has received the source packet successfully in the first slot are denoted as the candidate relays, the number of which is assumed to be $k(k=1,2 \cdots M)$. For one case, upon successful reception of the packet at d, it broadcasts an ACK message, and then s node transmits a new data packet in the next time slot and the candidate relays $r_{i}(i=1,2, \cdots, k)$ just idle. For the other case, if $d$ does not decode the packet correctly, it sends a NACK message that can be heard by the relays. If the first candidate relay can decode the source packet correctly in the first slot, it forwards the packet to $\mathrm{d}$ in the second slot. If $d$ does not receive correctly again, then it sends a NACK message and the second candidate relay, if it received the packet correctly in the first slot, forwards the source packet to the destination. This is repeated until $d$ gets the packet correctly or the $k$ trials corresponding to the $k$ candidate relays are exhausted.

Assuming the same channel model as the ISR system for the MIR system and using (4), the PER of the MIR system can be expressed by

$$
P E R^{M I R}=P E R_{s d} \prod_{i=1}^{M} P E R_{s i}+P E R_{s d} \sum_{k=1}^{M}\left\{\left(\begin{array}{c}
M \\
k
\end{array}\right)\left(\prod_{i=1}^{k}\left(1-P E R_{s i}\right) P E R_{i d}\right) \prod_{i=k+1}^{M} P E R_{s i}\right\}
$$

where the first tem represents the probability of the thing that the transmissions over the s-d channel and all the 
$\mathrm{s}-\mathrm{r}_{i}$ channels are failed and the second term stands for the probability of the thing that the transmission over the $\mathrm{s}-\mathrm{d}$ channel is failed while $k$ transmissions over the $\mathrm{s}-\mathrm{r}_{i}$ channel are successful, but, $k$ retransmissions are still failed.

In the MIR system, if the thing that $d$ receives the source packet correctly or the other thing that $d$ fails to receive the source packet and none of relays can receives the source packet correctly in the first slot happens, the packet transmission delay is one slot. In addition, the packet transmission delay is multiple time slots. Therefore, the normalized transmission delay of MIR system can be obtained as

$$
\begin{aligned}
T^{M I R} & =1 \cdot\left(1-P E R_{s d}+P E R_{s d} \prod_{i=1}^{M} P E R_{s i}\right) \\
& +\sum_{k=1}^{M}\left\{(k+1)\left(\begin{array}{c}
M \\
k
\end{array}\right)\left(\prod_{i=1}^{k}\left(1-P E R_{s i}\right)\right) \cdot \prod_{i=k+1}^{M} P E R_{s i}\left(\prod_{i=1}^{k-1}\left(P E R_{i d}\right)\right)\right\} \\
& +\sum_{k=1}^{M}\left\{\left(\begin{array}{c}
M \\
k
\end{array}\right)\left(\prod_{i=1}^{k}\left(1-P E R_{s i}\right)\right) \cdot \prod_{i=k+1}^{M} P E R_{s i}\left[\sum_{j=1}^{k-1}(j+1)\left(\prod_{i=1}^{j-1}\left(P E R_{i d}\right)\left(1-P E R_{j d}\right)\right)\right]\right\}
\end{aligned}
$$

Similarly to the ISR system, combining (15) and (16), the normalized throughput of the MIR system can be written as

$$
G^{M I R}=\frac{1-P E R^{M I R}}{T^{M I R}}
$$

Hence, the total consumed energy of transmitting one source packet with the MIR scheme can be expressed as

$$
\begin{aligned}
E^{M I R} & =\left(1-P E R_{s d}+P E R_{s d} \prod_{i=1}^{M} P E R_{s i}\right)\left(\left(P_{t}(1+\beta)+P_{c t}+2 P_{c r}\right) \frac{L}{R_{b}}\right) \\
& +\sum_{k=1}^{M}\left\{\left[\left((k+1) P_{t}(1+\beta)+(k+1) P_{c t}+(M+1+k) P_{c r}\right) \frac{L}{R_{b}}\right]\right. \\
& \left.\cdot\left(\begin{array}{c}
M \\
k
\end{array}\right)\left(\prod_{i=1}^{k}\left(1-P E R_{s i}\right)\right)\left(\prod_{i=k+1}^{M} P E R_{s i}\right) \prod_{i=1}^{k-1} P E R_{i d}\right\} \\
& +\sum_{k=1}^{M}\left\{\left(\begin{array}{c}
M \\
k
\end{array}\right)\left(\prod_{i=1}^{k}\left(1-P E R_{s i}\right)\right) \prod_{i=k+1}^{M} P E R_{s i}\right. \\
& \left.\cdot \sum_{j=1}^{k-1} \prod_{i=1}^{j-1}\left(P E R_{i d}\right)\left(1-P E R_{j d}\right)\left(\begin{array}{l}
(j+1) P_{t}(1+\beta)+(j+1) P_{c t}+ \\
(M+1+j) P_{c r}
\end{array}\right\} \frac{L}{R_{b}}\right\}
\end{aligned}
$$

Using (15) and (18), energy efficiency of the ISR system can be written as

$$
\eta^{M I R}=\frac{1-P E R^{M I R}}{E^{M I R}}
$$

\section{Simulation Results}

In this section, the performance of the ISR and MIR system with $M$ relays is compared through simulations. In all of the simulations, the system parameters take the following values when considered fixed: $\alpha=4, \beta=0.3, R_{b}$ $=20 \mathrm{k} \mathrm{bit} / \mathrm{s}, P_{t}=0.001 \mathrm{w}, P_{c t}=10^{-4} \mathrm{w}, P_{c r}=5 \times 10^{-5} \mathrm{w}, N_{0}=10^{-13.5}$.

The Figures 2-4 depict normalized throughput, normalized delay and energy efficiency performance of ISR and MIR systems for different values of the number of relays $(M)$ respectively. It can be seen from Figure 2 that normalized throughput of both ISR and MIR systems become larger and larger as the number of relays increases. If the number of relays is constant, normalized throughput of ISR system outperforms that of MIR system for any values of the distance of s-d link. This is because that multi-time retransmission of the MIR system results in that normalized delay of MIR system is higher than that of ISR system as shown in Figure 3. 


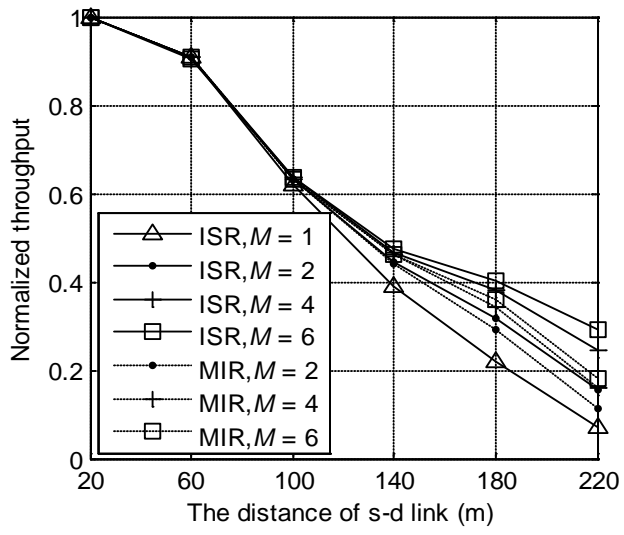

Figure 2. Normalized throughput versus the s-d distance for the ISR and MIR systems.

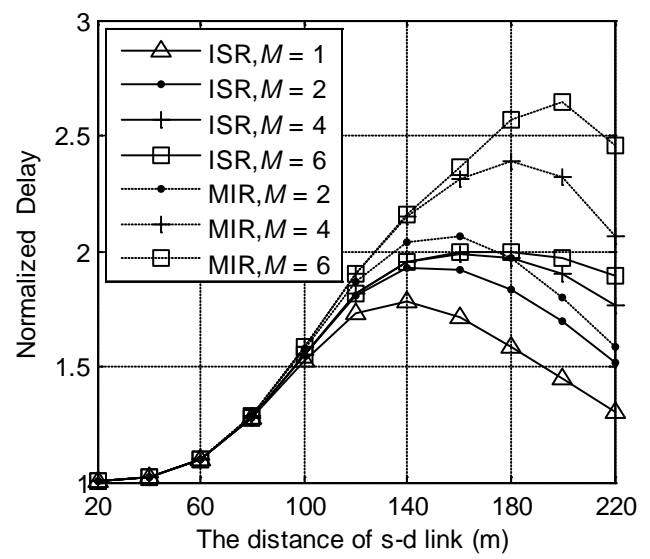

Figure 3. Normalized delay versus the s-d distance for the ISR and MIR systems.

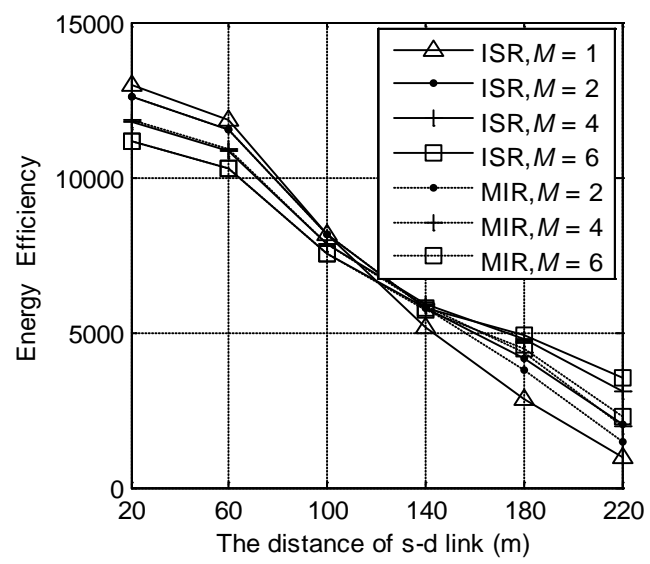

Figure 4. Energy efficiency versus the s-d distance for the ISR and MIR systems.

Given that the number of relays is constant, as shown in Figure 4, energy efficiency of ISR system outperforms that of MIR system. Because the implementation of ISR scheme requires maximum two time slots while MIR scheme might require more time slots, more energy for one same packet is consumed by MIR system.

Moreover, for the small value of the s-d distance, the system with one relay is more energy efficiency than the 
system multiple relays. But, for the large value of the s-d distance, the result is opposite. Hence, there are different the optimal number of relays which can maximize the energy efficiency of system for different values of the s-d distance.

We have conducted extensive simulation for ISR and MIR systems while the relays don't lie along a straight line and found that the performance of ISR system still outperforms that of MIR system and the foregoing conclusions don't change when the relays don't lie along a straight line.

\section{Conclusions and Future Work}

This paper proposes a new cooperative relaying scheme with best-relay selection termed as the incremental-selective relaying (ISR) for WSN. Expressions of normalized throughput, normalized delay and energy efficiency for the ISR and MIR system are derived respectively and their performance is compared through simulations. The results show that normalized throughput, normalized delay and energy efficiency for the ISR system all outperform the corresponding performances of the MIR system. Especially, there are different the optimal number of relays which can maximize the energy efficiency of system for different values of the s-d distance. The result can also be used to provide guidelines in determining the optimal number of relays for any given communication setup.

\section{Acknowledgements}

The research work was supported by National Science and Technology Major Project under Grant No. 2012ZX03006003. At last, the authors wish to thank the anonymous reviewers for their very helpful suggestions and comments.

\section{References}

[1] Akyildiz, I.F., Su, W., Sankarasubramaniam, Y. and Cayirci, E. (2002) A Survey on Sensor Networks. IEEE Commun. Mag., 40, 102-114. http://dx.doi.org/10.1109/MCOM.2002.1024422

[2] Song, L. and Hatzinakos, D. (2006) Cooperative Transmission in Poisson Distributed Wireless Sensor Networks: Protocol and Outage Probability. IEEE Trans. Wireless Commun., 5, 2834-2843. http://dx.doi.org/10.1109/TWC.2006.04598

[3] Sendonaris, A., Erkip, E. and Aazhang, B. (2003) User Cooperation Diversity-Part I: System Description. IEEE Trans. Commun., 51, 1927-1938. http://dx.doi.org/10.1109/TCOMM.2003.818096

[4] Sendonaris, A., Erkip, E. and Aazhang, B. (2003) User Cooperation Diversity-Part II: Implementation Aspects and Performance Analysis. IEEE Trans. Commun., 51, 1939-1948. http://dx.doi.org/10.1109/TCOMM.2003.819238

[5] Laneman, J.N., Wornell, G.W. and Tse, D.N.C. (2004) Cooperative Diversity in Wireless Networks: Efficient Protocols and Outage Behavior. IEEE Trans. Inform. Theory, 50, 3062-3080. http://dx.doi.org/10.1109/TIT.2004.838089

[6] Laneman, J.N. and Wornell, G.W. (2003) Distributed Space-Timecoded Protocols for Exploiting Cooperative Diversity in Wireless Networks. IEEE Trans. Inform. Theory, 49, 2415-2425. http://dx.doi.org/10.1109/TIT.2003.817829

[7] Boyer, J., Falconer, D.D. and Yanikomeroglu, H. (2004) Multinode Diversity in Wireless Relaying Channels. IEEE Trans. Commun., 52, 1820-1830. http://dx.doi.org/10.1109/TCOMM.2004.836447

[8] Sadek, A.K., Su, W. and Liu, K.J.R. (2007) Multinode Cooperative Communications in Wireless Networks. IEEE Trans. Signal Process., 55, 341-355. http://dx.doi.org/10.1109/TSP.2006.885773

[9] Cui, S., Goldsmith, A.J. and Bahai, A. (2004) Energy-Efficiency of MIMO and Cooperative MIMO Techniques in Sensor Networks. IEEE J. Select. Areas. Commun., 22, 1089-1098. http://dx.doi.org/10.1109/JSAC.2004.830916

[10] Jayaweera, S.K. (2006) Virtual MIMO-Based Cooperative Communication for Energy-Constrained Wireless Sensor Networks. IEEE Trans. Wireless Commun., 5, 984-989,. http://dx.doi.org/10.1109/TWC.2006.1633350

[11] Zhou, Z., Zhou, S., Cui, S. and Cui, J.-H. (1906) Energy-Efficient Cooperative Communication in Clustered Wireless Sensor Networks. Proc. IEEE Military Communications Conference, October 2006, 1-7. O. Wright and W. Wright, "Flying-Machine”, US Patent No. 821393. 